\title{
Revista Biomédica
}

http://revistabiomedica.mx

\section{Caso clínico}

\section{Tratamiento quirúrgico de la espondilitis tuberculosa (enfermedad de Pott). Reporte de un caso y revisión de la literatura.}

Nafxiel Jesús Brito-Núñez ${ }^{1 *}$; Luis Campos ${ }^{1}$; Francisco Díaz; Mariangela Velasquez; Edenys Izaguirre; Gustavo Faria-Méndez ${ }^{1}$; Cesar Roa-Chacón ${ }^{1}$; Aniello Romano ${ }^{1}$, María Lucy De Gouveia ${ }^{1,2}$; Magalys Tabasca $^{1,2}$, Jaime Krivoy ${ }^{1}$

1 Cátedra y servicio de neurocirugía. Hospital Universitario de Caracas, Caracas. Venezuela. 2 Escuela de medicina "Dr. Luis Razetti”. Universidad Central de Venezuela.

\section{ABSTRACT}

\section{Surgical treatment of tuberculous spondylitis (Pott's disease).} Case report and literature review.

Tuberculous spondylitis (Pott's disease) represent $1-5 \%$ of tuberculosis cases. We present a clinical case of a female patient of 14 years of age with tuberculous spondylitis (Pott's disease) complicated with vertebral body fracture T4, T7, T8 and T9, who underwent a posterior approach, bilateral complete laminectomy of T3, T4, T7, T8, T9 preserving the facet joints, more arthrodesis instrumented with sublaminar hooks in T1-T2 placement of sublaminar wires T5 and T6 placement of transpedicular screws in T11, and two titanium bars. With excellent postoperative results. In addition, the different surgical approaches to this pathology are discussed.

\section{RESUMEN}

La espondilitis tuberculosa (enfermedad de Pott) representa del $1-5 \%$ de los casos de tuberculosis. Se presenta un caso clínico de una paciente femenina de 14 años de edad con esta patología complicada con fractura de cuerpos vertebrales T4, T7, T8 y T9; a quien se le realizó un abordaje por vía posterior, consistente en laminectomía completa bilateral de T3, T4, T7, T8, T9 preservando las articulaciones facetarias, mas artrodesis instrumentada con ganchos sublaminares en
Historial del artículo

Recibido: 17 jun 2018

Aceptado: 26 nov 2018

Disponible online: 1 may 2019

\section{Palabras clave}

Enfermedad de Pott, laminectomía, tornillos pediculares, fractura patológica

\section{Keywords}

Pott's disease, laminectomy, pedicular screws, pathological fracture.

Copyright (C) 2019 por autores y Revista Biomédica.

Está trabajo esta licenciado bajo las atribuciones de la Creative Commons (CC BY).

http://creativecommons.org/licenses/by/4.0/

*Autor para correspondencia:

Nafxiel Jesús, Brito-Núñez, Cátedra y servicio de Neurocirugía. Ciudad Universitaria. Edificio. Hospital Universitario de Caraca. Caracas.

Venezuela

correo electrónico: nafxiel@gmail.com

htpp://revistabiomedica.mx. 
T1-T2, colocación de alambres sublaminares T5 y T6, tornillos transpediculares en T11 y dos barras de titanio. Con excelentes resultados postoperatorios. Además, se discuten las diferentes vías de abordaje quirúrgico de esta patología.

\section{INTRODUCCIÓN}

La tuberculosis (TBC) existe desde épocas milenarias y sigue siendo uno de los mayores problemas de salud pública a nivel global. Según datos de la Organización Mundial de la Salud (OMS) 2017, es causa de aproximadamente de 10 millones de muertes al año principalmente en relación con el VIH/SIDA $(1,6)$. De acuerdo a datos de la OMS En el año 2013 fueron notificados en Venezuela aproximadamente 6857 nuevos casos de tuberculosis, de los cuales 1163 fueron casos de TBC extrapulmonar y 461 (7\%) eran menores de 15 años. Para el año 2016 se vio un incremento del número de casos, cuando fueron notificados aproximadamente 8542 nuevos casos de tuberculosis, de los cuales $14 \%$ eran de TBC extrapulmonar y menos del $1 \%$ en menores de 15 años. (2).

La espondilitis tuberculosa, también llamada enfermedad de Pott, se define como una osteítis o caries de las vértebras, que suelen aparecer como una complicación de la tuberculosis de los pulmones, representa el 1-5\% de los casos y aproximadamente el $50 \%$ de la tuberculosis osteo-articular $(3,7)$. A pesar de todos los avances tecnológicos, el diagnóstico de la espondilitis tuberculosa sigue siendo un desafío, ya que depende de un alto grado de sospecha clínica. No obstante, la baja mortalidad reportada para ésta, aún se asocia con una morbilidad clínica significativa. Esta enfermedad consta de tres fases: de comienzo, de destrucción y de reparación, que conllevan una evolución clínica que, de ocurrir un retraso diagnóstico significativo, puede conducir a deformidades esqueléticas graves y complicaciones neurológicas irreversibles $(4,5,8)$. En este sentido, y considerando que se ha observado un incremento de la frecuencia enfermedad de Pott en Venezuela, el propósito del presente estudio es reportar un caso de TBC vertebral y revisar la literatura disponible haciendo énfasis en el manejo quirúrgico de esta patología, la importancia de su publicación radica en compartir los resultados obtenidos con el tratamiento quirúrgico.

\section{CASO CLÍNICO}

Femenino de 14 años de edad, quien refiere inicio de enfermedad actual nueve meses antes de su ingreso, caracterizado por dolor dorsolumbar, febrícula ocasional y diaforesis nocturnas, motivo por el cual acude a facultativo en pediatría quien evidencia esplenomegalia y refiere a hematólogo. En este servicio se indica tratamiento médico sin presentar mejoría clínica, por lo que al persistir el dolor es referida a especialista en reumatología quien indica estudios de laboratorios e imágenes. Dos semanas antes de su ingreso cae de su propia altura presentando paraparesia, motivo por el cual es remitida al servicio de neurocirugía del Hospital Universitario de Caracas donde se evalúa y se decide su ingreso. Entre sus antecedentes personales solamente se refiere hepatoesplenomegalia seis meses antes de su ingreso, niega alergia a medicamentos, sin contactos epidemiológicos de importancia.

Examen físico: Fuerza muscular $1 / 5$ en ambos miembros inferiores nivel de miotomo L2, hiperreflexia aquileana y patelar (III/IV), sensibilidad superficial y profunda conservada, sin trastornos esfinterianos. Se realizan paraclínicos en sangre que reportaron para su ingreso, leucocitosis (11.200 leucocitos por mm3) con ligera neutrofilia, velocidad de sedimentación globular (VSG) $20 \mathrm{~mm}$ en una hora (considerado normal de acuerdo a la referencia del laboratorio), proteína $C$ reactiva (PCR) elevada 6 veces su valor normal, PPD positivo, resto dentro de límites normales. La serología para virus de inmunodeficiencia humana resultó negativa.

El estudio de tomografía computarizada (TC) de columna vertebral mostró lesiones erosivas (geodas) y fractura de los cuerpos vertebrales en el segmento T3, T4, T7, T8 y T9 con compromiso de la altura de los cuerpos vertebrales afectados y otra geoda en cuerpo vertebral de L2 (Figura 1).

El estudio de resonancia magnética $(\mathrm{RM})$ de columna vertebral reveló lesiones múltiples de ubicación extradural en T3, T4, T7, T8 y 


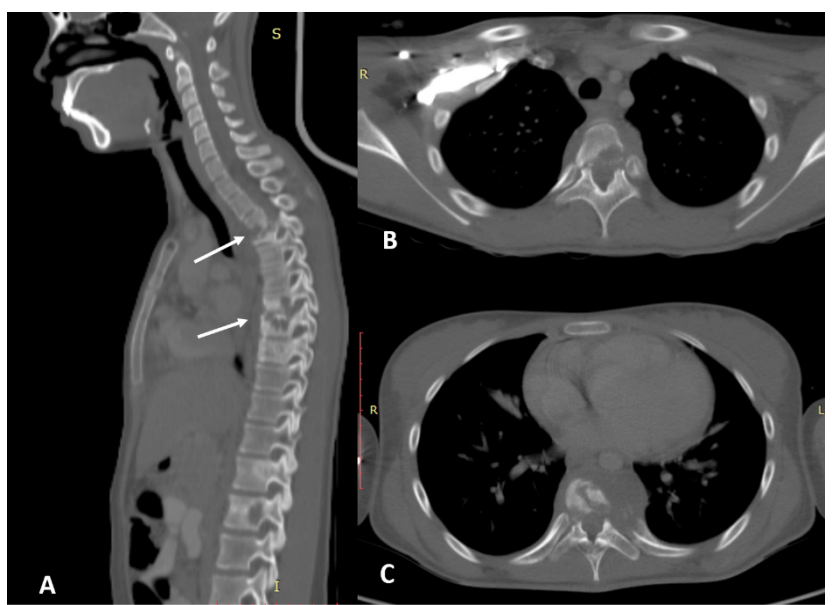

Figura 1. Tomografía de columna vertebral preoperatorias. A: Corte sagital. B: corte axial en T3 y T4. C: Corte axial T8. Observe las lesiones erosivas (geodas) y fractura de los cuerpos vertebrales en el segmento T3, T4, T7, T8 y T9.

T9, hipointensas en la secuencia T1 y T2 con relación al líquido cefalorraquídeo, que ocupa partes blandas anteriores adyacentes y cuerpos vertebrales de T3, T4, T7, T8 y T9, que abarca además pedículos $\mathrm{y}$ elementos posteriores de dichas vertebras que condiciona reducción del canal medular y compresión anterior de la médula y colapso de cuerpos vertebrales de T3, T4, T7 y $\mathrm{T} 8$, con disminución de la altura de los mismos y aplastamiento de discos intervertebrales de T3-T4 y T7-T8 (Figura 2).

Finalmente, los estudios radiográficos de tórax y la tomografía de tórax no evidenciaron lesiones. La paciente ingresó al servicio de neurocirugía con los siguientes diagnósticos: 1. Síndrome de motoneurona superior secundario a compresión medular por lesión ocupante del espacio en T3 secundarios a: 1.1 Fracturas patológicas de T3T4, T7-T8 y 1.2 Enfermedad de Pott T3, T4, T7, T8 y T9, y 2. Síndrome de compresión medular. Para su manejo, la paciente fue llevada a cirugía y mediante abordaje por vía posterior, se le practicó laminectomía completa bilateral de T3, T4, T7, T8, T9 preservando las articulaciones facetarias, más artrodesis instrumentada con ganchos sublaminares en T1-T2, colocación de alambres sublaminares en T5 y T6, colocación de tornillos transpediculares en T11, más dos barras de titanio. Se toman muestras

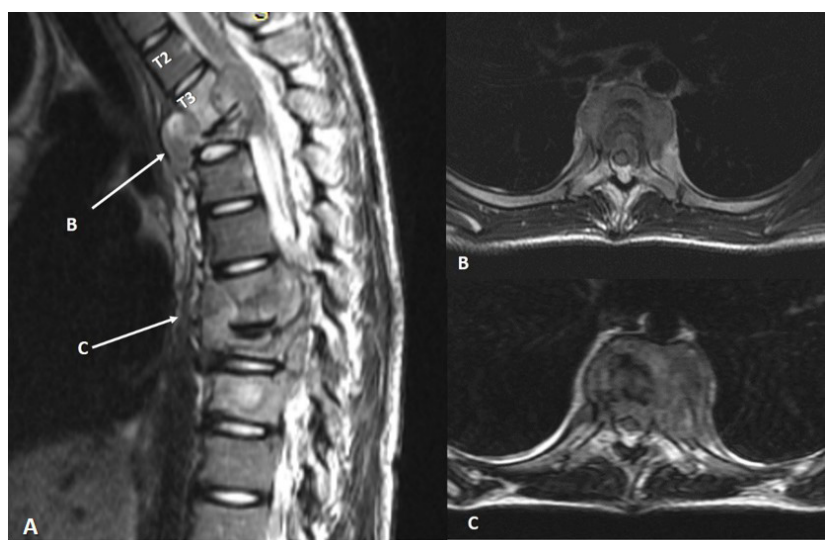

Figura 2. Resonancia magnética (RM) de columna dorsal. A: Corte sagital. B: Corte axial en T4. C: Corte axial T7. Se observan lesiones múltiples de ubicación extradural en T3, T4, $\mathrm{T} 7$, T8 y T9, que abarca elementos posteriores de dichas vertebras con invasión de canal medular y compresión anterior de la médula, además se observa colapso de cuerpos vertebrales de T3, T4, T7 y T8, con disminución de la altura de los mismos, y aplastamiento de discos intervertebrales de T3-T4 y T7-T8.

para biopsia y cultivo para micobacterias de lesión blanco-grisácea de aspecto grumoso. (Figura 3).

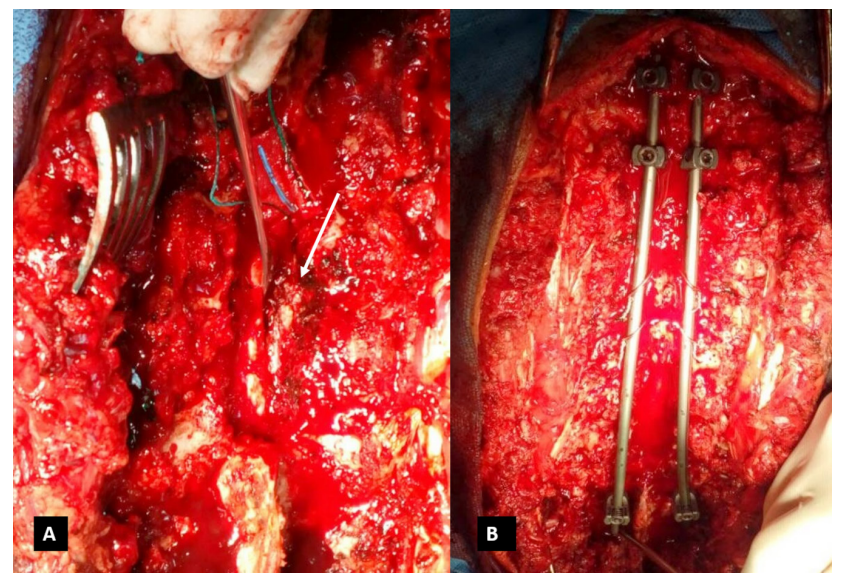

Figura 3. Imágenes del transoperatorio. Lesión delimitada por el instrumento y la flecha (A). Artrodesis instrumentada (B).

En el postoperatorio inmediato la paciente presenta disnea y tos, con matidez a la percusión y abolición de murmullo vesicular de hemitórax derecho, con lo que se plantea derrame pleural que ameritó colocación de tubo de tórax, evidenciando la presencia de exudado lechoso, fue valorada por servicio de neumología quien indica iniciar tratamiento con rifampicina, etambutol e isoniazida. 
El estudio de rayos $\mathrm{X}$ de tórax post-toracotomía muestra derrame pleural y presencia de la instrumentación (Figura 4).

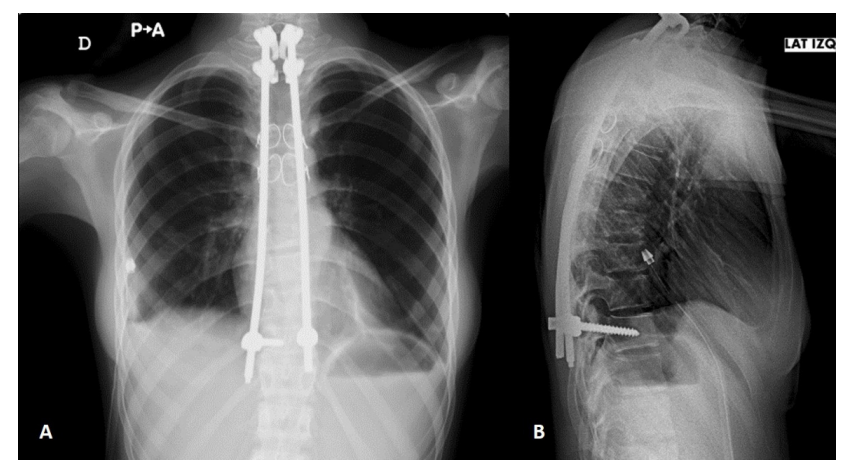

Figura 4. Radiografía de anteroposterior (A) y lateral (B) de tórax posoperatoria. Observe la disposición del material de osteosíntesis.

El reporte de anatomía patológica en los preparados histológicos con coloración de hematoxilina y eosina, mostró abundante infiltrado inflamatorio de linfocitos, plasmocitos y ocasionales polimorfonucleares con abundantes células gigantes de Langerhans y extensas zonas de necrosis, hay tejido fibroblástico con proliferación de vasos sanguíneos de pequeño tamaño, sin evidencia de agentes infecciosos, atipias ni neoplasias. concluyendo inflamación crónica granulomatosa necrotizante. El cultivo con la tinción de Ziehl Neelsen reportó presencia de bacilos ácido alcohol resistentes, confirmando el diagnóstico de la enfermedad de Pott.

La tomografía tomada en el postoperatorio evidenció restitución de la altura de cuerpos vertebrales (Figura 5) y reducción de la angulación relativa del plano sagital preoperatoria $\mathrm{y}$ posoperatoria de $36,5^{\circ}$ a $26,1^{\circ}$. La paciente mejora clínicamente y fue dada de alta. Actualmente, su evolución clínica es satisfactoria dado que recupera la fuerza muscular, deambula sin ayuda y realiza sus actividades cotidianas.

\section{DISCUSIÓN}

La espondilitis tuberculosa ha afectado a la humanidad desde tiempos de los reyes de Egipto (3400 aC). Hipócrates, considerado el padre de la

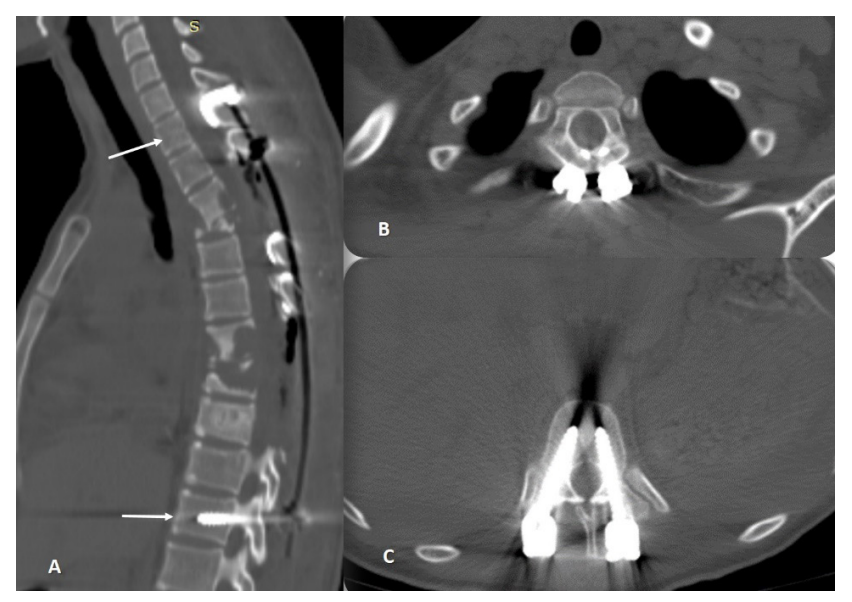

Figura 5. Tomografía posoperatoria. A: corte sagital observe el restablecimiento de la altura del cuerpo vertebral. B: corte axial en T2 a nivel observe la disposición del gancho sublaminar. C: Corte axial en T11, observe la disposición de los tornillos transpediculares.

cirugía de columna vertebral (100-300 aC), describió la tuberculosis espinal y la deformidad gibosa tuberculosa en su trabajo sobre articulaciones. Muchos siglos después, en 1779, Sir Percival Pott describió la enfermedad tuberculosa de la columna vertebral y sus secuelas de paraplejia como "ese tipo de parálisis de las extremidades inferiores que con frecuencia acompaña a la curvatura de la columna vertebral". Pott también describió la clásica tríada clínica de la tuberculosis espinal (cifosis, abscesos y paraplejía), que luego se denominó criterio diagnóstico de tuberculosis (6).

La espondilitis tuberculosa es más frecuente en varones adultos. Suele diseminarse por vía hematógena desde el sistema respiratorio o genitourinario, aunque también pueden colonizar la columna por extensión directa. Afecta por orden de frecuencia a la columna dorsal, lumbar y cervical (8). Clínicamente se manifiesta con dolor en un $83-100 \%$ de los casos y síntomas constitucionales, incluida la fiebre y diaforesis nocturna (9) como en el caso presentado. Las deformidades de la columna vertebral y el déficit neurológico son las peores complicaciones presentes en la espondilitis tuberculosa hasta en un $45 \%$, según lo reportado (10). De acuerdo a una casuística publicada recientemente la prevalencia de espondilitis tuberculosa es notablemente más alta en pacientes 
portadores del virus de inmunodeficiencia humana representando un $76 \%$ de los casos (11).

En cuanto a los métodos diagnósticos de la enfermedad de Pott, clásicamente las imágenes de la TBC espinal demuestran la enfermedad del cuerpo vertebral en las vértebras contiguas. La extensión subligamentosa del absceso debajo del ligamento longitudinal anterior o posterior a una vértebra adyacente es un hallazgo clásico. El disco intervertebral se involucra a medida que la enfermedad progresa. En la enfermedad avanzada, hay colapso de los elementos espinales y acuñamiento anterior que conduce a una angulación severa y formación de gibas (12), el diagnóstico es altamente presuntivo en paciente con clínica parecida a la presentada por esta paciente. La RM es el estudio de imagen de elección donde el hallazgo más frecuente es la espondilolistesis, reducción de los espacios en el disco en otros segmentos, erosiones irregulares y destrucción focal en placas de los extremos adyacentes del músculo psoas. También se mencionan la presencia de una colección epidural anterior como un signo de enfermedad activa (24) y la confirmación bacteriológica.

En el manejo de la enfermedad de Pott se ha demostrado que el drenaje percutáneo guiado por TC combinado con la cirugía de abordaje posterior es seguro y eficaz para el tratamiento de la tuberculosis espinal dorsal y lumbar con enormes abscesos ilio-psoas en adultos (13). Sin embargo, aún existe la controversia en relación con el tratamiento quirúrgico, la técnica de desbridamiento y artrodesis, bien sea por vía anterior vs vía posterior vs combinación anterior y posterior, que se describen a continuación. El tratamiento clásico de la espondilitis tuberculosa consiste en desbridamiento anterior e injerto estructural con cresta iliaca, peroné o costilla conocida como técnica de Hong Kong, sin embargo, estos injertos pueden sufrir fracturas, deslizamiento y deformidad $(8,14)$. El desbridamiento anterior radical seguido de la reconstrucción con injerto estructural clásicamente se ha mencionado que es el patrón de oro del tratamiento quirúrgico de la enfermedad de Pott (8). Jin et al, estudiaron 23 pacientes con tuberculosis de la columna toracolumbar en fase de destrucción realizando desbridamiento radical, autoinjerto estructural e instrumentación anterior con placas y tornillos, recibieron tratamiento antituberculoso por nueve meses y utilizaron corset por seis meses, concluyendo que el autoinjerto e instrumentación intercorporales anterior en una etapa muestran más ventajas en pacientes seleccionados porque pueden proporcionar suficiente estabilidad segmentaria a la columna vertebral afectada, una buena corrección de la deformidad cifótica y la promoción de la fusión ósea. En combinación con la quimioterapia estándar, la reconstrucción temprana de la estabilidad espinal juega un papel importante en el tratamiento quirúrgico de la tuberculosis espinal. La quimioterapia regular y el mantenimiento de la estabilidad espinal son la estrategia principal para el tratamiento de cualquier tuberculosis espinal y no se pueden separar (15), sin embargo, se han notificado las complicaciones inherentes al tratamiento como lo son ruptura pleural intraoperatoria, lesión de los nervios, lesiones vasculares, tromboembolismos pulmonares después de la cirugía, íleo paralítico en incluso diabetes insípida (16).

En pacientes en fases destructivas iniciales, con cifosis leve o en aquellos muy debilitados, una alternativa de tratamiento es la descompresión posterior (laminectomía) asociada a una instrumentación transpedicular con artrodesis posterolateral, en una única intervención $(17,18)$ similar al caso presentado. Gao et al (2017), realizaron abordaje por vía posterior en 54 pacientes con esta patología y compararon dos técnicas, cesta intersomáticadetitaniomástornillos transpediculares, dos niveles por encima y dos por debajo de la lesión y otro grupo con injerto autólogo de cresta iliaca más tronillos transpediculares, encontrando que, a los nueve meses de evolución, en todos los casos, hubo fusión de los cuerpos vertebrales (19). Cui et al (2016) estudiaron 81 pacientes con enfermedad de Pott en la columna torácica que se sometieron a instrumentación anterior o posterior combinada con diferentes métodos de desbridamiento, la descompresión y fusión del injerto, dio como resultado que la instrumentación posterior fue 


\section{Brito-Núñez, et al}

más efectiva que la anterior en la corrección de la cifosis y el mantenimiento de la corrección (20). En otro estudio multicéntrico, realizado por $\mathrm{Wu}$ et al (2018), del tratamiento quirúrgico de la tuberculosis de la columna torácica concluyó que la fijación posterior fue superior a la anterior en la corrección de la cifosis y el mantenimiento de la estabilidad espinal. La cirugía posterior en un solo paso puede lograr la misma eficacia que la cirugía sólo ésta o combinada, con menos trauma y pérdida de sangre, y tiempos quirúrgicos más cortos. Sin embargo, para lesiones anchas o abscesos paraespinales, destrucción ósea severa y defectos de columna anterior y media que son demasiado grandes, después del desbridamiento como para requerir un injerto óseo de segmento largo, el abordaje anterior combinado es indispensable (21). En un estudio bibliométrico publicado recientemente, que incluyó del año 2000 a 2016, realizado por Fisahn et al (2017) recomendó el abordaje quirúrgico anterior para la columna cervical y posterior para la columna torácica y lumbar. El combinado (anterior más posterior) preferentemente en los casos pediátricos. El grado de corrección de la deformidad, los resultados neurológicos y la fusión forman las bases principales para evaluar los resultados quirúrgicos (22). Por lo que la individualización de cada caso es fundamental, similar al caso presentado cuyo abordaje fue por vía posterior obteniendo buenos resultados en el postoperatorio.

En resumen, la tuberculosis toracolumbar puede resolverse de manera adecuada mediante abordaje anterior o posterior en una etapa, con desbridamiento, injerto óseo y fijación interna, siendo el posterior el que tiene ventajas de menor traumatismo y una mejor corrección de la deformidad (23).

\section{REFERENCIAS}

1. Global tuberculosis report 2017. Geneva: World Health Organization; 2017. Licence: CC BY-NCSA 3.0 IGO. [citado, 11 de febrero 2018] Disponible en: http://apps. who.int/iris/bitstream/10665/259366/1/9789241565516eng.pdf?ua $=1$

2. World Health Organization. Tuberculosis [Internet]. Venezuela (Bolivarian Republic of): WHO; 2016. [cited 12 Feb 2018] Available from: https://extranet.who.int/ $\underline{\text { sree/Reports?op=Replet\&name }=/ \text { WHO HQ Reports/ }}$ $\underline{\text { G2/PROD/EXT/TBCountryProfile\&ISO2=ve\&outtype }}$ $=$ pdf

3. Peto H. M., Pratt R. H., Harrington T. A., LoBue P. A., Armstrong L. R. Epidemiology of extrapulmonary tuberculosis in the United States, 1993-2006. Clinical Infectious Diseases. 2009;49(9):1350-1357. DOI: https://doi.org/10.1086/605559.

4. Pellisé F. Tuberculosis and Pott's disease, still very relevant health problems. European Spine Journal. 2013;22(4):527-528. DOI: https://doi.org/10.1007/ S00586-012-2531-5.

5. Lacerda C, Linhas R, Duarte R. Tuberculous Spondylitis: A Report of Different Clinical Scenarios and Literature Update. Case Rep Med. 2017; 2017: 4165301. DOI: https://doi.org/10.1155/2017/4165301

6. Rajasekaran S, Kanna RM, Shetty AP. History of spine surgery for tuberculous spondylodiscitis. Unfallchirurg. 2015 Dec;118 Suppl 1:19-27. DOI: https://doi. org/10.1007/s00113-015-0093-9.

7. Trecarichi EM, Di Meco E, Mazzotta V, Fantoni M. Tuberculous spondylodiscitis: epidemiology, clinical features, treatment, and outcome. Eur Rev Med Pharmacol Sci. 2012 Apr;16 Suppl 2:58-72.

8. Ballesteros R, Gómez E, Bonsfills N, González R, García FJ, Moreno J, et al. Columna Toracolumbar. 1 era edición. Madrid, España. Edit. MARBAN LIBROS. 2012, Pp. 566-572.

9. Pigrau-Serrallach C, Rodríguez-Pardo D. "Bone and joint tuberculosis," European Spine Journal. 2013: 22(4):556-566.

10. Jain AK. Tuberculosis of the spine: a fresh look at an old disease. Journal of Bone and Joint Surgery. 2010; 92(7): 905-913.

11. Marais S, Roos I, Mitha A, Mabusha SJ, Patel V, Bhigjee AI. Spinal tuberculosis: Clinicoradiological findings in 274 patients. Clin Infect Dis. 2018 Jan 11. DOI: https:// doi.org/10.1093/cid/ciy020

12. Cantwell LM, Perkins JC, Keyes DCV. Pott's disease in a patient with subtle red flags. J Em Med. DOI: https:// doi.org/10.1016/j.jemermed.2017.12.007

13. Zou DX, Zhou JL, Zhou XX, Jiang XB. Clinical efficacy of CT-guided percutaneous huge ilio-psoas abscesses drainage combined with posterior approach surgery for the management of dorsal and lumbar spinal tuberculosis in adults. Orthop Traumatol Surg Res. 2017 Dec;103(8):1251-1255. DOI: https://doi.org/10.1016/j. otsr.2017.07.015.

14. Hodgson AR, Stock FE, Fang HS, Ong GB. Anterior spinal fusion. The operative approach and pathological findings in 412 patients with Pott's disease of the spine. Br J Surg. 1960 Sep;48:172-8.

15. Jin D1, Qu D, Chen J, Zhang H. One-stage anterior interbody autografting and instrumentation in primary surgical management of thoracolumbar spinal tuberculosis. 
Eur Spine J. 2004 Mar;13(2):114-21.

16. Fu ZQ, Zhang ZM, Jin DD, Chen JT, Qu DB. [Complications of the anterior surgical approach for thoracolumbar spine tuberculosis: causes and countermeasures]. Nan Fang Yi Ke Da Xue Xue Bao. 2009 Jun;29(6):1229-31.

17. Lee SH, Sung JK, Park YM. Single-stage transpedicular decompression and posterior instrumentation in treatment of thoracic and thoracolumbar spinal tuberculosis: a retrospective case series. J Spinal Disord Tech. 2006 Dec;19(8):595-602.

18. Shen X, Huang X, Xiao S, Liu H, Zhang Y, Xiang T, et al. Surgical treatment of selected patients with multilevel contiguous thoracolumbar spinal tuberculosis by only posterior instrumentation without any bone fusion. Int J Clin Exp Med. 2015 Oct 15;8(10):18611-9.

19. Gao Y, Ou Y, Deng Q, He B, Du X, Li J. Comparison between titanium mesh and autogenous iliac bone graft to restore vertebral height through posterior approach for the treatment of thoracic and lumbar spinal tuberculosis. PLoS ONE 2017; 12(4): e0175567. DOI: https://doi. org/10.1371/journal.pone.0175567

20. Cui X, Li LT, Ma YZ. Anterior and Posterior Instrumentation with Different Debridement and Grafting
Procedures for Multi-Level Contiguous Thoracic Spinal Tuberculosis. Orthop Surg. 2016 Nov;8(4):454-461. DOI: https://doi.org/10.1111/os.12288.

21. Wu W, Lyu J, Liu X, Luo F, Hou T, Zhou Q. Surgical Treatment of Thoracic Spinal Tuberculosis: A Multicenter Retrospective Study. World Neurosurg. 2018 Feb;110:e842-e850. DOI: https://doi.org/10.1016/j. wneu.2017.11.126.

22. Fisahn C, Alonso F, Hasan GA, Tubbs RS, Dettori JR, Schildhauer TA, Rustagi T. Trends in Spinal Surgery for Pott's Disease (2000-2016): An Overview and Bibliometric Study. Global Spine J. 2017 Dec;7(8):821828. DOI: https://doi.org/10.1177/2192568217735827.

23. Lin B, Dai LL, Chen ZD, Zheng Y. Comparision of clinical effects between one-stage anterior and posterior approach in treating thoracolumbar tuberculosis with debridement and internal fixation. Zhongguo $\mathrm{Gu}$ Shang. 2017 Sep 25;30(9):792-798. DOI: https://doi. org/10.3969/j.issn.1003-0034.2017.09.003.

24. Narayan V, Mohammed N, Savardekar AR, Patra DP, Nanda A. Tuberculous Spondylolisthesis: A Reappraisal of the Clinicoradiologic Spectrum and Surgical Treatment Paradigm. World Neurosurg. 2018 Jun;114:361-367. DOI: https://doi.org/10.1016/j.wneu.2018.02.157. 